\title{
Mosquito Control Based on Pesticides and Endosymbiotic Bacterium Wolbachia
}

\author{
Linchao $\mathrm{Hu}^{1,2} \cdot$ Cui Yang ${ }^{3}$ Yuanxian Hui ${ }^{1,2} \cdot$ Jianshe $\mathrm{Yu}^{1}$ \\ Received: 10 December 2020 / Accepted: 28 February 2021 / Published online: 13 April 2021 \\ (c) The Author(s) 2021
}

\begin{abstract}
Mosquito-borne diseases, such as dengue fever and Zika, have posed a serious threat to human health around the world. Controlling vector mosquitoes is an effective method to prevent these diseases. Spraying pesticides has been the main approach of reducing mosquito population, but it is not a sustainable solution due to the growing insecticide resistance. One promising complementary method is the release of Wolbachia-infected mosquitoes into wild mosquito populations, which has been proven to be a novel and environment-friendly way for mosquito control. In this paper, we incorporate consideration of releasing infected sterile mosquitoes and spraying pesticides to aim to reduce wild mosquito populations based on the population replacement model. We present the estimations for the number of wild mosquitoes or infection density in a normal environment and then discuss how to offset the effect of the heatwave, which can cause infected mosquitoes to lose Wolbachia infection. Finally, we give the waiting time to suppress wild mosquito population to a given threshold size by numerical simulations.
\end{abstract}

Keywords Wolbachia - Mosquito-borne diseases · Population replacement · Population suppression $\cdot$ Pesticides $\cdot$ Heatwave

$凶 \quad$ Jianshe $\mathrm{Yu}$

jsyu@gzhu.edu.cn

1 Center for Applied Mathematics, Guangzhou University, Guangzhou 510006, People's Republic of China

2 College of Mathematics and Information Sciences, Guangzhou University, Guangzhou 510006, People's Republic of China

3 The Second Affiliated Hospital of Guangzhou Medical University, Guangzhou 510260, People's Republic of China 


\section{Introduction}

Mosquito-borne diseases, such as dengue fever and Zika, have posed a serious threat to the health of human beings around the world and bring a great financial burden to the governments in the tropic and sub-tropic areas (Kyle and Harris 2008; Rasmussen et al. 2016). Since there are no efficient vaccines available, controlling the vector population is the most effective measure of preventing mosquito-borne diseases. For a long time, vector control methods have mainly relied on the extensive use of insecticides. Although the utilization of insecticides reduces the mosquito population size greatly, it causes environmental pollution and offers only a short-term solution due to the mosquito resistance to insecticides (Kyle and Harris 2008; Ooi et al. 2006). An innovative and environmentally friendly strategy for the control of mosquito-borne diseases is to employ the maternally inherited endosymbiotic bacterium Wolbachia, whose infection in Aedes mosquitoes can reduce their transmission potential to spread viruses (Bian et al. 2010; Dutra et al. 2016). In addition, Wolbachia induces cytoplasmic incompatibility (CI) that causes early embryonic death when Wolbachia-infected males mate with uninfected females (Laven 1956), resulting in the decrease of the proportion of uninfected mosquitoes. Therefore, we can release infected mosquitoes to invade and replace the wild population (population replacement) or suppress wild mosquito population to reduce mosquito bites (population suppression). There has been an increasing interest toward the spreading dynamics of Wolbachia; see (Farkas and Hinow 2010; Hu et al. 2019; Keeling et al. 2003; Shi and Yu 2020; Yu and Zheng 2019) for the theoretical works on population replacement and (Huang et al. 2018, 2020; Yu 2018; Zhang et al. 2020) on population suppression. By releasing infected mosquitoes twice a week, our team, led by Xi, eradicated more than $90 \%$ of Aedes albopictus in an island in South Guangzhou (Zheng et al. 2019), which verifies the feasibility of mosquito suppression in the field. On the other hand, it is reported in Nature News that releasing Wolbachia-infected mosquitoes in Yogyakarta reduces $77 \%$ of dengue cases compared with areas that did not receive infected mosquitoes (Callaway 2020). The regression model in Ryan et al. (2019) also showed a $96 \%$ reduction in dengue incidence in Wolbachia-treated populations. These trials proved that population replacement based on Wolbachia may greatly block the transmission of mosquito-borne diseases.

Suggested by the empirical data (McMeniman et al. 2009; Walker et al. 2011; Yeap et al. 2011), we give three basic assumptions: perfect maternal transmission, complete $\mathrm{CI}$, and equal sex determination. Motivated by the work in Yu (2018), let $b_{I}$ and $b_{U}$ be the total numbers of offspring per unit of time, per infected and uninfected mosquitoes, respectively. Let $\delta_{I}$ and $\delta_{U}$ denote the density-independent decay rates of infected and uninfected mosquitoes, and $d_{I}$ and $d_{U}$ the density-dependent decay rates of infected and uninfected mosquitoes, respectively. Denote by $x(t)$ and $y(t)$ the numbers of infected and uninfected mosquitoes, respectively. Then we obtain the following differential equation model to characterize the dynamics of infected and 
uninfected mosquitoes,

$$
\left\{\begin{array}{l}
\frac{\mathrm{d} x}{\mathrm{~d} t}=\left(b_{I}-\delta_{I}\right) x-d_{I} x(x+y) \\
\frac{\mathrm{d} y}{\mathrm{~d} t}=b_{U} y \frac{y}{x+y}-\delta_{U} y-d_{U} y(x+y)
\end{array}\right.
$$

The term $y /(x+y)$ represents is the probability of mating with wild mosquitoes. Since the infected or uninfected mosquitoes don't die out naturally in the wild, we assume

$$
b_{I}>d_{I}+\delta_{I}, \quad b_{U}>d_{U}+\delta_{U} .
$$

In general, Wolbachia infections bring fitness cost to their hosts such as reduced fecundity or longevity (McMeniman et al. 2009; Walker et al. 2011; Weeks et al. 2002). Here we consider these differences and ignore the diversity of density-dependent death rates between infected and uninfected mosquitoes based on Zhang et al. (2015). Then we assume that

$$
b_{U} \geq b_{I}, \quad \delta_{I} \geq \delta_{U} \quad \text { and } \quad d_{I}=d_{U}=d .
$$

Most of the existing literatures discuss population replacement or population repression separately. In this work, we consider subsequent release of infected sterile mosquitoes and spraying insecticides based on the population replacement model (1). Let $R(t)$ be the release abundance of infected mosquitoes at time $t$. Let $\phi_{I}(t)$ and $\phi_{U}(t)$ denote the excess death rates caused by pesticides for infected and uninfected mosquitoes, respectively. Then we obtain the following model by combining the use of pesticides and the release of infected mosquitoes:

$$
\left\{\begin{array}{l}
\frac{\mathrm{d} x}{\mathrm{~d} t}=\left(b_{I}-\delta_{I}-\phi_{I}(t)\right) x-d x(x+y) \\
\frac{\mathrm{d} y}{\mathrm{~d} t}=b_{U} y \frac{y}{x+y+R(t)}-\left(\delta_{U}+\phi_{U}(t)\right) y-d y(x+y) .
\end{array}\right.
$$

Recently, scientists found that infected mosquitoes may lose Wolbachia at egg and larvae stages due to the strike of heatwaves (Ross et al. 2020, 2017). This leakage situation has been discussed in (Farkas and Hinow 2010; Keeling et al. 2003; Zheng et al. 2018). Let $\mu$ denote the fraction of uninfected offspring produced by infected mosquitoes. By reconsidering the numbers of new born offspring of both uninfected and infected mosquitoes based on (4), we obtain an improved model in Sect. 3.

Some mosquito-borne diseases occur periodically and are triggered by imported patients, for example the dengue fever in Guangzhou. It requires emergency measures when the dengue cases in the neighboring areas are large. The empirical data in Guangzhou show that the wild mosquito population must be reduced to a low level such that the Breteau index is less that 5 to prevent dengue fever (Duan et al. 2009). Then we can estimate a safe threshold number $\mathcal{S}$ of wild mosquitoes as suppression goal. In $\mathrm{Hu}$ et al. (2015), Hu et al. (2019), we discussed the sufficient conditions for Wolbachia fixation in deterministic or stochastic environment. In this study, we continue 
to investigate the detailed dynamical behavior of the wild mosquitoes and estimate the time required (waiting time) to reduce wild mosquitoes to a level below $\mathcal{S}$. With the help of numerical simulations, we see that the combination of population replacement and suppression can greatly improve the control speed of wild mosquitoes.

In this work, we start in Sect. 2 with an ordinary differential equation model for mosquito population replacement. By introducing suppression measures, we can accelerate the reduction speed of wild population and we present estimations of the wild mosquito abundance or infection density defined by $p(t)=x(t) /(x(t)+y(t))$. In Sect. 3 , we consider a special environmental condition in which the infected mosquitoes may lay uninfected eggs due to the heatwaves. We discuss how to offset this negative effect by releasing infected sterile mosquitoes or spraying pesticides. Finally, we discuss the waiting time to reduce wild mosquitoes to a level below a given threshold in Sect. 4, and shows the requirement for release abundance or spraying density of pesticides for given parameters and initial state.

\section{Mosquito Control in Normal Environment}

In this part, we introduce two control measures and their combination based on population replacement model (1). According to the assumptions (2) and (3), system (1) admits three equilibria (See Fig.1): two locally stable equilibria $E_{1}\left(0, \frac{b_{U}-\delta_{U}}{d}\right), E_{2}\left(\frac{b_{I}-\delta_{I}}{d}, 0\right)$, and a saddle point

$$
E_{3}\left(\frac{\left(b_{U}-\delta_{U}-b_{I}+\delta_{I}\right)\left(b_{I}-\delta_{I}\right)}{b_{U} d}, \frac{\left(\delta_{U}+b_{I}-\delta_{I}\right)\left(b_{I}-\delta_{I}\right)}{b_{U} d}\right) .
$$

The dynamic behaviors of (1) are similar to the cases in (Farkas and Hinow 2010; Keeling et al. 2003; Zheng et al. 2014). $E_{1}$ and $E_{2}$ are local stable and stay on the $y$-axis and $x$-axis, respectively. $E_{3}$ is a saddle point in the first quadrant. There exists a separatrix $\mathcal{H}: y=h(x)$ in the first quadrant below which the number of Wolbachia-infected mosquitoes declines to zero and above which the Wolbachia-infected mosquitoes spread to the whole population.

Define the infection density by $p(t)=x(t) /(x(t)+y(t))$. The infection density is easier to monitor than the detailed wild mosquito abundance. Many works discussed the existence of the threshold $p^{*}$ (Caspari and Watson 1959; Hu et al. 2015; Zheng et al. 2014): the initial infection frequency $p_{0}>p^{*}$ leads to Wolbachia fixation, while $p_{0}<p^{*}$ leads to Wolbachia extinction. It follows from (1) that

$$
\begin{aligned}
\frac{\mathrm{d} p(t)}{\mathrm{d} t} & =\frac{x^{\prime} y-x y^{\prime}}{(x+y)^{2}} \\
& =\frac{x y}{(x+y)^{2}}\left(b_{I}-\delta_{I}+\delta_{U}-b_{U} \frac{y}{x+y}\right) \\
& =p(t)(1-p(t))\left(b_{I}-\delta_{I}+\delta_{U}-b_{U}(1-p)\right)
\end{aligned}
$$




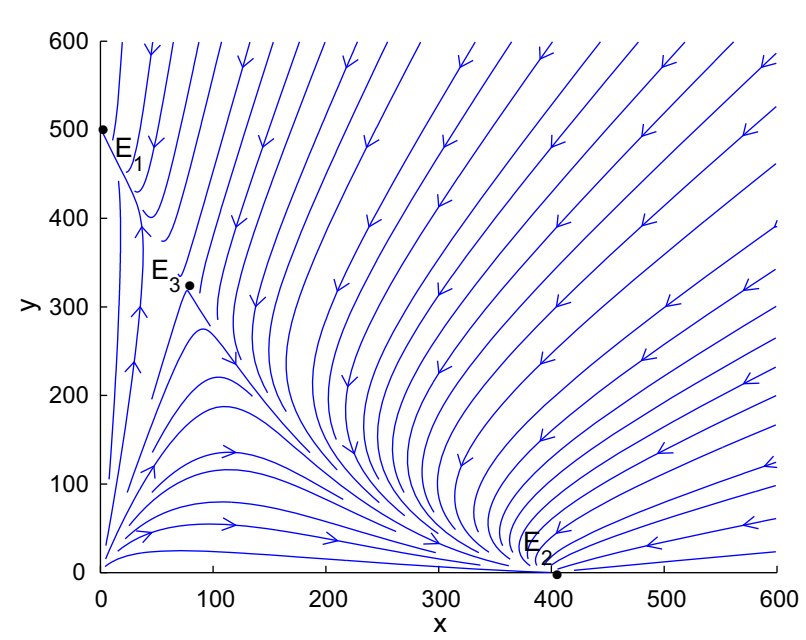

Fig. 1 The vector field direction of system (1). Let $b_{I}=0.45, b_{U}=0.55, \delta_{I}=0.05, \delta_{U}=0.048$, $d=0.001$. System (1) admits three equilibria: $E_{1}$ and $E_{2}$ are local stable, and they stay on the $y$-axis and $x$-axis, respectively; $E_{3}$ is a saddle point in the first quadrant

$$
=b_{U} p(t)(1-p(t))\left(p(t)-\left(1-\frac{b_{I}-\delta_{I}+\delta_{U}}{b_{U}}\right)\right) \text {. }
$$

Clearly, $p(t)$ increases in $t$ and approaches 1 if $1-\frac{b_{I}-\delta_{I}+\delta_{U}}{b_{U}}<p(0)<1$, decreases in $t$ and approaches 0 if $0<p(0)<1-\frac{b_{I}-\delta_{I}+\delta_{U}}{b_{U}}$. Thus $1-\frac{b_{I}-\delta_{I}+\delta_{U}}{b_{U}}$ is a threshold value for the initial infection density above which the Wolbachia will invade to the mosquito population successfully, and below which Wolbachia frequency declines to zero. Note that $p(t)=1-\left(b_{I}-\delta_{I}+\delta_{U}\right) / b_{U}$ implies $y(t) / x(t)=\left(b_{I}+\delta_{U}-\right.$ $\left.\delta_{I}\right) /\left(b_{U}-b_{I}+\delta_{I}-\delta_{U}\right)$. Then

$$
\mathcal{H}: y=h(x)=\frac{b_{I}+\delta_{U}-\delta_{I}}{b_{U}-b_{I}+\delta_{I}-\delta_{U}} x
$$

is the separatrix which divides the first quadrant into two parts, the upper one is the basin of attraction of $E_{1}$ and the lower one is the basin of attraction of $E_{2}$. Providing that the initial release ensures successful invasion of Wolbachia-infected mosquitoes, we focus on how to reduce wild mosquitoes to a safe level within a given time.

\subsection{Repeated Release of Infected Sterile Mosquitoes.}

Here we consider repeated release of infected sterile mosquitoes based on population replacement model (1). Then (4) is reduced to

$$
\left\{\begin{array}{l}
\frac{\mathrm{d} x}{\mathrm{~d} t}=\left(b_{I}-\delta_{I}\right) x-d x(x+y) \\
\frac{\mathrm{d} y}{\mathrm{~d} t}=b_{U} y \frac{y}{x+y+R(t)}-\delta_{U} y-d y(x+y) .
\end{array}\right.
$$


We first consider the ratio of released mosquito abundance to wild mosquito abundance. Define the release ratio

$$
\mathcal{K}(t):=R(t) /(x(t)+y(t)) .
$$

$\mathcal{K}(t)=0$ corresponding to the case $R(t)=0$. As it is difficult to fix the release ratio to a constant, we let $\mathcal{K}_{1}$ and $\mathcal{K}_{2}$ be the lower and upper bound of $\mathcal{K}(t)$, respectively, i.e.,

$$
\mathcal{K}_{1}<\mathcal{K}(t)<\mathcal{K}_{2}
$$

Since $\frac{y}{x+y+R(t)}=\frac{1}{1+\mathcal{K}(t)} \cdot \frac{y}{x+y}$, we rewrite (7) as

$$
\left\{\begin{array}{l}
\frac{\mathrm{d} x}{\mathrm{~d} t}=\left(b_{I}-\delta_{I}\right) x-d x(x+y), \\
\frac{\mathrm{d} y}{\mathrm{~d} t}=b_{U} y \cdot \frac{1}{1+\mathcal{K}(t)} \cdot \frac{y}{x+y}-\delta_{U} y-d y(x+y) .
\end{array}\right.
$$

Define

$$
\bar{b}_{U}=b_{U} /\left(1+\mathcal{K}_{2}\right) \text { and } \hat{b}_{U}=b_{U} /\left(1+\mathcal{K}_{1}\right)
$$

It follows from (8) that $\bar{b}_{U}<b_{U} /(1+\mathcal{K}(t))<\hat{b}_{U}$. We next compare system (9) with the following two systems:

$$
\begin{aligned}
& \left\{\begin{array}{l}
\frac{\mathrm{d} x}{\mathrm{~d} t}=\left(b_{I}-\delta_{I}\right) x-d x(x+y), \\
\frac{\mathrm{d} y}{\mathrm{~d} t}=\bar{b}_{U} y \frac{y}{x+y}-\delta_{U} y-d y(x+y) .
\end{array}\right. \\
& \left\{\begin{array}{l}
\frac{\mathrm{d} x}{\mathrm{~d} t}=\left(b_{I}-\delta_{I}\right) x-d x(x+y), \\
\frac{\mathrm{d} y}{\mathrm{~d} t}=\hat{b}_{U} y \frac{y}{x+y}-\delta_{U} y-d y(x+y) .
\end{array}\right.
\end{aligned}
$$

Theorem 1 Let $\left(x_{0}, y_{0}\right)$ be an initial state with which the solution of $(1)$ approaches $E_{2}$. Assume $\bar{b}_{U}<\hat{b}_{U}$. Let $(X(t), Y(t)),\left(X_{1}(t), Y_{1}(t)\right)$ and $\left(X_{2}(t), Y_{2}(t)\right)$ denote the solutions of (7), (11) and (12) initiating from $\left(x_{0}, y_{0}\right)$, respectively. Then $Y(t) \rightarrow 0$ as $t \rightarrow \infty$ and $Y_{1}(t) \leq Y(t) \leq Y_{2}(t)$ for all $t \geq 0$.

Proof Let $p(t), p_{1}(t)$ and $p_{2}(t)$ denote the infection densities of systems (7), (11) and (12) at the initial state $\left(x_{0}, y_{0}\right)$, respectively. We first show that $p_{1}(t) \leq p(t) \leq p_{2}(t)$. By (5) we have

$$
\begin{aligned}
& \frac{d p_{1}(t)}{d t}=p_{1}(t)\left(1-p_{1}(t)\right)\left(b_{I}-\delta_{I}+\delta_{U}-\bar{b}_{U}\left(1-p_{1}\right)\right) \\
& \frac{d p_{2}(t)}{d t}=p_{2}(t)\left(1-p_{2}(t)\right)\left(b_{I}-\delta_{I}+\delta_{U}-\hat{b}_{U}\left(1-p_{2}\right)\right) .
\end{aligned}
$$


A

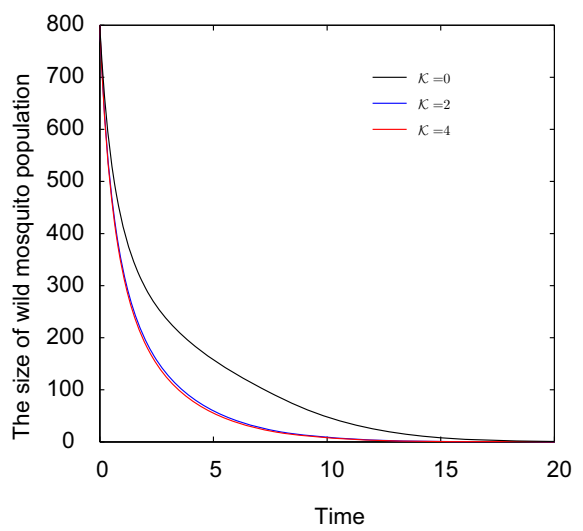

B

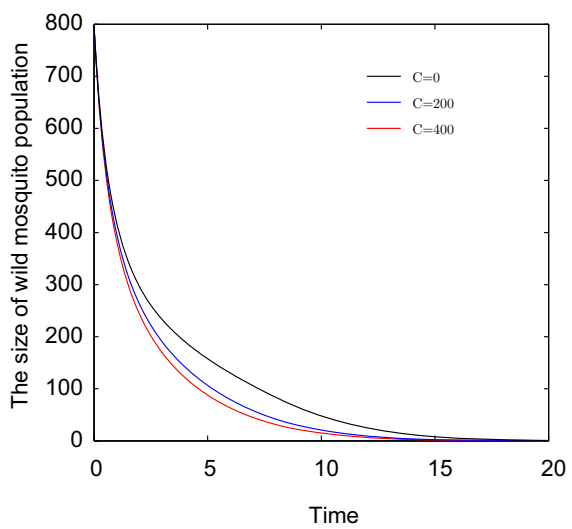

Fig. 2 The declines of wild mosquito abundance under different release strategies. Let $b_{I}=0.45, b_{U}=$ $0.55, \delta_{I}=0.05, \delta_{U}=0.048, d=0.001$. Set the initial state $\left(x_{0}, y_{0}\right)=(500,800)$. Panel a shows the number of wild mosquitoes changes with time $t$ in different release ratios. Panel b shows the number of wild mosquitoes decreases with time $t$ in different constant release amounts (Color figure online)

Then both $p_{1}(t)$ and $p_{2}(t)$ increase in $t$ from the initial infection density $x_{0} /\left(x_{0}+y_{0}\right)$. Since $\bar{b}_{U}<\hat{b}_{U}$, we find that $\left.\frac{d p_{1}(t)}{d t}\right|_{\left(x_{0}, y_{0}\right)}>\left.\frac{d p_{2}(t)}{d t}\right|_{\left(x_{0}, y_{0}\right)}$, and there exists $t_{1}>0$ such that $p_{1}(t)>p_{2}(t)$ for $0 \leq t \leq t_{1}$. If $p_{1}(t)<p_{2}(t)$ for some $t>t_{1}$, we set $t_{2}=\inf \left\{t \mid p_{1}(t)<p_{2}(t)\right\}$. Then $p_{1}\left(t_{2}\right)=p_{2}\left(t_{2}\right)$ and $\frac{d p_{1}(t)}{d t} \leq \frac{d p_{2}(t)}{d t}$ at the intersection. However, by the expressions of $\frac{d p_{1}}{d t}$ and $\frac{d p_{2}}{d t}$ we get that $\frac{d p_{1}(t)}{d t}>\frac{d p_{2}(t)}{d t}$ at any point in the first quadrant, which gives a contradiction. Thus $p_{1}(t)>p_{2}(t)$ for all $t>0$. By using the same idea to compare $p(t)$ with $p_{1}(t)$ and $p_{2}(t)$, respectively, we can derive that $p_{1}(t) \geq p(t) \geq p_{2}(t)$ for $t>0$.

Now we prove $Y_{1}(t) \leq Y_{2}(t)$ for $t \geq 0$. By (11) and (12) we see that $\frac{d Y_{1}(t)}{d t}<\frac{d Y_{2}(t)}{d t}$ at the initial state $\left(x_{0}, y_{0}\right)$. Then there exists $t_{3}$ such that $Y_{1}(t)<Y_{2}(t)$ for $0 \leq t \leq t_{3}$. If $Y_{1}(t)>Y_{2}(t)$ for some $t>t_{3}$, we set $t_{4}=\inf \left\{t \mid Y_{1}(t)>Y_{2}(t)\right\}$. Then $Y_{1}\left(t_{4}\right)=$ $Y_{2}\left(t_{4}\right)$ and $\frac{d Y_{1}(t)}{d t}>\frac{d Y_{2}(t)}{d t}$ at the intersection. It follows from $p_{1}\left(t_{4}\right) \geq p_{2}\left(t_{4}\right)$ and $Y_{1}\left(t_{4}\right)=Y_{2}\left(t_{4}\right)$ that $X_{1}\left(t_{4}\right) \geq X_{2}\left(t_{4}\right)$. Then by the expressions of $\frac{d Y_{1}}{d t}$ and $\frac{d Y_{2}}{d t}$ in (11) and (12), we derive $\frac{d Y_{1}\left(t_{4}\right)}{d t}<\frac{d Y_{2}\left(t_{4}\right)}{d t}$, which contradicts the assumption $Y_{1}(t)>Y_{2}(t)$ for some $t>t_{3}$. Thus $Y_{1}(t)<Y_{2}(t)$. By comparing $Y(t)$ with $Y_{1}(t)$ and $Y_{2}(t)$, respectively, we obtain that $Y_{1}(t) \leq Y(t) \leq Y_{2}(t)$ for all $t \geq 0$. Since $Y_{1}(t) \rightarrow 0$ and $Y_{2}(t) \rightarrow 0$ as $t \rightarrow \infty$, we have $Y(t) \rightarrow 0$ as $t \rightarrow \infty$

Figure 2a shows that if the release ratio $2<\mathcal{K}(t)<4$, then the curve for wild mosquito abundance lies in the very narrow area sandwiched by the red and blue curves. Another common release strategy is to release infected mosquitoes by a compensation policy such that the loss of infected mosquitoes is compensated by new releasing, then 
we assume $R(t)=C$ is a constant function for $t>0 \mathrm{Yu}$ (2018). In this case, we have

$$
\left\{\begin{array}{l}
\frac{\mathrm{d} x}{\mathrm{~d} t}=\left(b_{I}-\delta_{I}\right) x-d x(x+y), \\
\frac{\mathrm{d} y}{\mathrm{~d} t}=b_{U} y \frac{y}{x+y+C}-\delta_{U} y-d y(x+y) .
\end{array}\right.
$$

The discussions in Theorem 1 are applicable for system (13). Figure 2B shows the number of wild mosquitoes decreases with $t$ under different constant release amount. If $200<C<400$, the curve of wild mosquito abundance lies in the narrow area sandwiched by the blue and red curves.

\subsection{Spray Pesticides}

Here we consider the use of pesticides based on population replacement model (1). Then model (4) is reduced to

$$
\left\{\begin{array}{l}
\frac{\mathrm{d} x}{\mathrm{~d} t}=\left(b_{I}-\delta_{I}-\phi_{I}(t)\right) x-d x(x+y) \\
\frac{\mathrm{d} y}{\mathrm{~d} t}=b_{U} y \frac{y}{x+y}-\left(\delta_{U}+\phi_{U}(t)\right) y-d y(x+y)
\end{array}\right.
$$

It is natural to assume that $\phi_{I}(t)$ and $\phi_{U}(t)$ increase or decrease simultaneously. Define

$$
a(t)=b_{I}-\delta_{I}-\phi_{I}(t)+\delta_{U}+\phi_{U}(t)
$$

Then the infection density can be expressed by

$$
\frac{d p(t)}{d t}=p(t)(1-p(t))\left(a(t)-b_{U}(1-p)\right)
$$

By (16), we obtain that $p(t)$ increases in $t$ if the initial infection density $p_{0}=\frac{x_{0}}{x_{0}+y_{0}}$ satisfies

$$
\min \{a(t)\}>b_{U}\left(1-p_{0}\right), \text { or equivalently, } p_{0}>1-\frac{\min \{a(t)\}}{b_{U}} .
$$

Assume that $\phi_{U}(t)-\phi_{I}(t)$ takes the maximum value and the minimum value at time $t_{1}^{*}$ and $t_{2}^{*}$, respectively. Then

$$
\max \{a(t)\}=a\left(t_{1}^{*}\right) \text { and } \min \{a(t)\}=a\left(t_{2}^{*}\right) .
$$

Define

$$
\hat{\phi}_{U}=\phi_{U}\left(t_{1}^{*}\right), \quad \bar{\phi}_{U}=\phi_{U}\left(t_{2}^{*}\right), \quad \hat{\phi}_{I}=\phi_{I}\left(t_{1}^{*}\right) \text { and } \bar{\phi}_{I}=\phi_{I}\left(t_{2}^{*}\right) .
$$


Construct the following systems

$$
\begin{aligned}
& \left\{\begin{array}{l}
\frac{\mathrm{d} x}{\mathrm{~d} t}=\left(b_{I}-\delta_{I}-\bar{\phi}_{I}\right) x-d x(x+y), \\
\frac{\mathrm{d} y}{\mathrm{~d} t}=b_{U} y \frac{y}{x+y}-\left(\delta_{U}+\bar{\phi}_{U}\right) y-d y(x+y) .
\end{array}\right. \\
& \left\{\begin{array}{l}
\frac{\mathrm{d} x}{\mathrm{~d} t}=\left(b_{I}-\delta_{I}-\hat{\phi}_{I}\right) x-d x(x+y), \\
\frac{\mathrm{d} y}{\mathrm{~d} t}=b_{U} y \frac{y}{x+y}-\left(\delta_{U}+\hat{\phi}_{U}\right) y-d y(x+y) .
\end{array}\right.
\end{aligned}
$$

Remark 2.1 Suppose that (17) hold. Let $p(t), p_{1}(t)$ and $p_{2}(t)$ denote the infection densities of (7), (20) and (21) at the initial state $\left(x_{0}, y_{0}\right)$, respectively. As spraying pesticides only affects the term $a(t)$ in (16), the density infection of system (7) with the measure of spraying pesticides satisfies $p_{1}(t)<p(t)<p_{2}(t)$ for all $t \geq 0$.

From (5), we see that $p(t)$ is not affected by the use of insecticides if $\phi_{I}(t) \equiv \phi_{U}(t)$.

Remark 2.2 Suppose that (17) hold and $\phi_{I}(t) \equiv \phi_{U}(t)$. Redefine $\bar{\phi}_{I}=\min \left\{\phi_{I}(t)\right\}$, $\bar{\phi}_{U}=\min \left\{\phi_{U}(t)\right\}$ in (20) and $\hat{\phi}_{I}=\max \left\{\phi_{I}(t)\right\}, \hat{\phi}_{U}=\max \left\{\phi_{U}(t)\right\}$ in (21). Let $(X(t), Y(t)),\left(X_{1}(t), Y_{1}(t)\right)$ and $\left(X_{2}(t), Y_{2}(t)\right)$ denote the solutions of (7), (20) and (21) initiating from $\left(x_{0}, y_{0}\right)$, respectively. Then we have $Y(t) \rightarrow 0$ as $t \rightarrow \infty$ and $Y_{1}(t) \leq Y(t) \leq Y_{2}(t)$ for all $t \geq 0$ by the same method in Theorem 1 .

When $\phi_{I}(t)=\phi_{U}(t)=\phi(t)$, we use an example to show the estimations of $\phi(t)$ and its effect on mosquito control. Assume that pesticides are sprayed every 7 days and the residual effects last for $T_{1}$ days with $3<T_{1}<4$. Assume that $\phi(t)$ is sandwiched by $y=y_{1}(t)$ and $y=y_{2}(t)$ with

$y_{1}=\left\{\begin{array}{c}-\frac{0.3}{4}(t-4), \quad n T<t<n T+4, \\ 0, \quad n T+4<t<(n+1) T,\end{array} \quad y_{2}=\left\{\begin{array}{c}-\frac{0.2}{3}(t-3), \quad n T<t<n T+3, \\ 0, \quad n T+3<t<(n+1) T,\end{array}\right.\right.$

$n=0,1,2, \cdots$ (See Fig. 3A). Let $b_{I}=0.45, b_{U}=0.55, \delta_{I}=0.05, \delta_{U}=0.048$, $d=0.001$ and $T=7$. The actual curve of wild mosquito abundance under the measure of spraying pesticides is sandwiched by the blue and red curves in Fig. 3B, which represent the cases that $\phi(t)=y_{1}(t)$ and $\phi(t)=y_{1}(t)$, respectively.

Remark 2.3 When $\phi_{I}(t) \not \equiv \phi_{U}(t)$, the relation among $Y(t), Y_{1}(t)$ and $Y_{2}(t)$ is uncertain. Although the use of pesticides increases the death rate of wild mosquitoes, it may slow down the decline of the wild mosquito population if the damage of insecticides to infected mosquitoes is greater than that to uninfected mosquitoes (See Fig. 4).

\subsection{Combine the Release of Infected Sterile Mosquitoes and Spraying Pesticides.}

In this part, we discuss model (4). Recalling the definitions of $a(t)$ and $b_{U}(t)$ in (10), (15) and (18), we have

$$
\frac{d p(t)}{d t}=p(t)(1-p(t))\left(a(t)-b_{U}(t)(1-p)\right),
$$


A

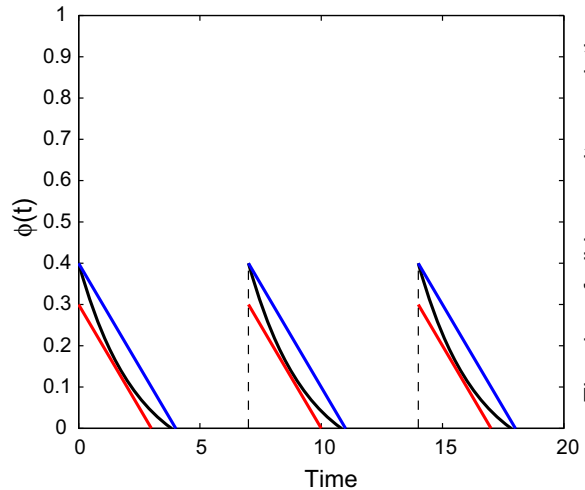

B

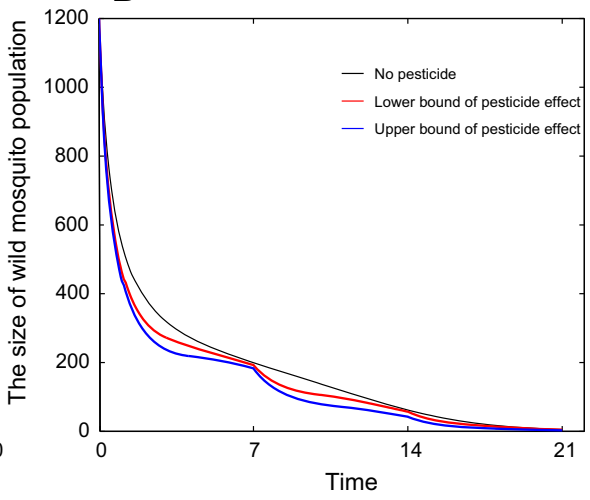

Fig. 3 Estimations of $\phi(t)$ and its effect on mosquito control. a Let $T=7$. The black curve shows that $\phi(t)$ decreases with time after each spraying. The blue segments and red segments are the upper and lower bounds of $\phi(t)$, respectively. b Let $\left(x_{0}, y_{0}\right)=(500,1200)$ and the parameters be the same as in Fig. 2 . The black curve shows the number of wild mosquitoes decreases without spraying pesticides, and the blue and red curves show the numbers of wild mosquitoes decrease with time when $\phi(t)=y_{1}(t)$ and $\phi(t)=y_{2}(t)$, respectively. The actual curve of wild mosquito abundance is sandwiched by the blue curve and red curve (Color figure online)

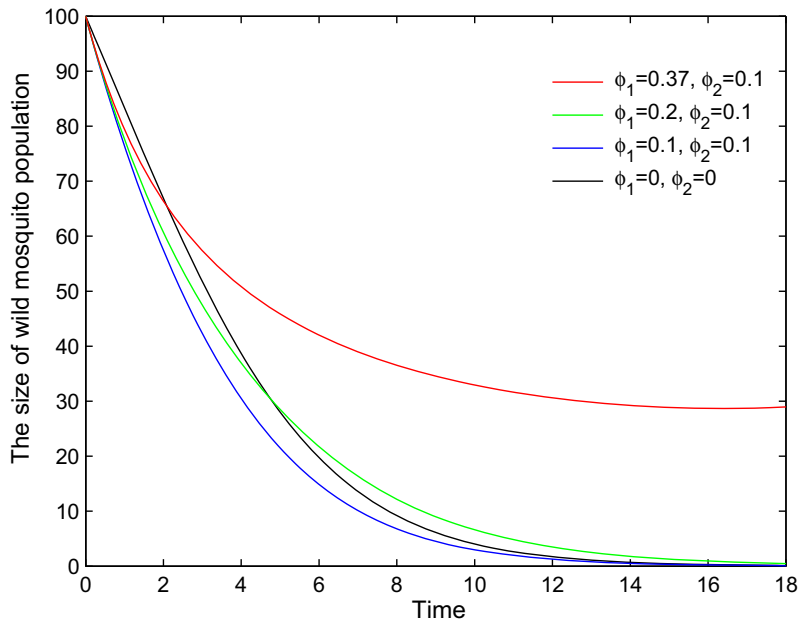

Fig. 4 Pesticides may slow down the decline of the wild mosquito abundance. Let $b_{I}=0.45, b_{U}=0.55$, $\delta_{I}=0.05, \delta_{U}=0.048, d=0.001$ and $\left(x_{0}, y_{0}\right)=(200,100)$. The black curve shows the number of wild mosquitoes changes with time $t$ without spraying pesticides. If $\phi_{I}=\phi_{U}=0.1$, the blue curve shows wild mosquitoes decreases faster than the previous case. If we keep $\phi_{U}=0.1$ and increase $\phi_{I}$ to 0.2 , the green curve shows the abundance of wild mosquitoes decreases faster than no-pesticide case at first, but it decreases slower after a while. If we continue to increase $\phi_{I}$ to 0.37 without changing $\phi_{U}$, the red curve shows the wild mosquitoes cannot be eradicated (Color figure online) 
where $\bar{a}=a\left(t_{1}^{*}\right) \leq a(t) \leq a\left(t_{2}^{*}\right)=\hat{a}$ and $\bar{b}_{U} \leq b_{U}(t) \leq \hat{b}_{U}$. Construct the following systems

$$
\begin{aligned}
& \frac{d \bar{p}(t)}{d t}=\bar{p}(t)(1-\bar{p}(t))\left(\bar{a}-\hat{b}_{U}(1-\bar{p}(t))\right), \\
& \frac{d \hat{p}(t)}{d t}=\hat{p}(t)(1-\hat{p}(t))\left(\hat{a}-\bar{b}_{U}(1-\hat{p}(t))\right) .
\end{aligned}
$$

Remark 2.4 Let the initial state $\left(x_{0}, y_{0}\right)$ satisfies $p(0)=x_{0} /\left(x_{0}+y_{0}\right)>1-\bar{a} / \hat{b}$. By the similar comparison method in Theorem 1 we can obtain $\bar{p}(t) \leq p(t) \leq \hat{p}(t)$.

Now we consider the case that to release infected mosquitoes and to spray pesticides separately, and the release ratio and spraying density are fixed. Let $t_{0}, t_{1}, t_{2}, \cdots$ be the switch times between the two stages. Then the durations in all stages are $\left[t_{0}, t_{1}\right)$, $\left[t_{1}, t_{2}\right),\left[t_{2}, t_{3}\right), \cdots$. In view of the discussion in Remark 2.3, we assume $\phi_{I}(t)<\phi_{U}(t)$. Redefine $\hat{b}_{U}=b_{U}$ and $\hat{a}=b_{I}-\delta_{I}-\phi_{I}(t)+\delta_{U}+\phi_{U}(t)$ in spraying stage, and $\bar{b}_{U}=b_{U} /(1+\mathcal{K}(t))$ and $\bar{a}=b_{I}-\delta_{I}+\delta_{U}$ in releasing stage. Then $\bar{a}<\hat{a}$ and $\bar{b}_{U}<\hat{b}_{U}$. Construct systems

$$
\begin{aligned}
& \frac{d p_{1}(t)}{d t}=p_{1}(t)\left(1-p_{1}(t)\right)\left(\hat{a}-\hat{b}_{U}\left(1-p_{1}(t)\right)\right) \\
& \frac{d p_{2}(t)}{d t}=p_{2}(t)\left(1-p_{2}(t)\right)\left(\bar{a}-\bar{b}_{U}\left(1-p_{2}(t)\right)\right) .
\end{aligned}
$$

Let $p(t)$ denote the Wolbachia infection density of systems (22). Define

$$
\beta_{1}=1-\hat{a} / \hat{b}_{U}, \quad \beta_{2}=1-\bar{a} / \bar{b}_{U}, \quad p_{1}^{*}=\min \left\{\beta_{1}, \beta_{2}\right\}, \quad p_{2}^{*}=\max \left\{\beta_{1}, \beta_{2}\right\} .(26)
$$

Then we have the following estimations for $p(t)$.

Theorem 2 Suppose that $p(0)>p_{2}^{*}, \bar{b}_{U}<\hat{b}_{U}$ and $\bar{a}<\hat{a}$. Let system (22) switches between systems (24) and (25) and the staying times in the two systems are $T_{1}$ and $T_{2}$, respectively. If $T_{1}$ and $T_{2}$ satisfy $0<\bar{T}_{1}<T_{1}<\hat{T}_{1}<\infty$ and $0<\bar{T}_{2}<T_{2}<\hat{T}_{2}<$ $\infty$, then we have the following result:

(1) If $p(0) \geq \beta_{3}=1-(\hat{a}-\bar{a}) /\left(\hat{b}_{U}-\bar{b}_{U}\right)$, then $p_{2}(t) \leq p(t) \leq p_{1}(t)$ for all $t>0$.

(2) If $0<p(0)<\beta_{3}$, then there exists a $t^{*}>0$ such that $p_{1}(t)<p_{2}(t)$ when $0<t<t^{*}$, and $p_{1}(t)>p_{2}(t)$ when $t>t^{*}$. Denote by $\mathcal{D}_{1}$ the area enclosed by $p_{1}(t)$ and $p_{2}(t)$ for $t>t^{*}$. Then $p=p(t)$ enters $\mathcal{D}_{1}$ for sufficiently large $t$ and stays in this area thereafter.

Proof (1) Since $p_{1}(0)=p_{2}(0)=p(0)>p_{2}^{*}$, all the three functions $p, p_{1}$, and $p_{2}$ increase in $t>0$. Define

$$
g_{0}(p)=\hat{a}-\hat{b}_{U}+\hat{b}_{U} p-\left(\bar{a}-\bar{b}_{U}+\bar{b}_{U} p\right)
$$


It can be easily verified that

$$
p>\beta_{3} \quad \text { if and only if } \quad g_{0}(p)>0 .
$$

Hence at $t=0$, it holds that

$$
p_{1}^{\prime}(0)-p_{2}^{\prime}(0)=p(0)(1-p(0)) g_{0}(p(0))>0 \text {. }
$$

Therefore, $p_{1}(t)>p_{2}(t)$ for small $t>0$. Indeed, this relation remains valid for all $t>0$; otherwise, there would be some $\tau>0$ such that $p_{1}(t)>p_{2}(t)$ for $0<t<\tau$, but $p_{1}(\tau)=p_{2}(\tau)$. Hence $p_{1}^{\prime}(\tau) \leq p_{2}^{\prime}(\tau)$, which contradicts the fact that (29) is still valid if $p(0)$ is replaced with $p_{1}(\tau)$. It follows that $p_{1}(t)>p_{2}(t)$ for all $t>0$. Define

$$
r(t)=\ln \frac{p(t)}{1-p(t)} .
$$

To confirm the relation of $p(t)$ with $p_{1}(t)$ and $p_{2}(t)$, we denote by $r_{1}(t)$ and $r_{2}(t)$ the corresponding forms of $r(t)$ defined in (30) where $p$ is replaced by $p_{1}$ or $p_{2}$. Due to the monotonic dependance of $r(t)$ on $p(t), p(t) \leq p_{1}(t)$ is equivalent to $r(t) \leq r_{1}(t)$. Suppose for contradiction that $r(t)>r_{1}(t)$ at some $t$. Then

$$
\hat{t}=\inf \left\{t \mid r(t)>r_{1}(t)\right\}
$$

is finite. We claim that $p(t)$ is governed by system (25) at time $\hat{t}$. If this is not true, then there exists an $\epsilon>0$ such that the system stays in System (24) for $t \in[\hat{t}, \hat{t}+\epsilon)$, and therefore, $r(t)=r_{1}(t)$ in this interval, which contradicts the definition of $\hat{t}$. We now show that the system does not stay in System (25) at time $\hat{t}$ either. If it does, then by (31) and $r_{1}(0)=r(0)$ we find that $r_{1}(\hat{t})=r(\hat{t})$ and so the right-hand derivative of $r(t)$ must not be less than that of $r_{1}(t)$ at $\hat{t}$, i.e., $r_{+}^{\prime}(\hat{t}) \geq r_{1+}^{\prime}(\hat{t})$. In addition, by taking derivatives of $r_{1}(t)$ and $r_{2}(t)$, we find

$$
r_{1}^{\prime}(t)=\hat{a}-\hat{b}_{U}+\hat{b}_{U} p_{1}(t) \text { and } r_{2}^{\prime}(t)=\bar{a}-\bar{b}_{U}+\bar{b}_{U} p_{2}(t)
$$

It then follows that

$$
r_{1+}^{\prime}(\hat{t})-r_{+}^{\prime}(\hat{t})=\hat{a}-\hat{b}_{U}+\hat{b}_{U} p_{1}(\hat{t})-\left(\bar{a}-\bar{b}_{U}+\bar{b}_{U} p_{1}(\hat{t})\right)=g_{0}(\hat{t})>0,
$$

which gives a contradiction. Thus

$$
r(t) \leq r_{1}(t) \text { and } p(t) \leq p_{1}(t)
$$

for all $t>0$. The same reasoning shows that $p(t) \geq p_{2}(t)$.

(2) Define

$$
s(t)=r_{1}(t)-r_{2}(t)
$$


Clearly, $s(0)=0$. Since $p(0)<\beta_{3}$, we have

$$
s^{\prime}(0)=\hat{a}-\hat{b}_{U}+\hat{b}_{U} p(0)-\left(\bar{a}-\bar{b}_{U}+\bar{b}_{U} p(0)\right)=g_{0}(0)<0 .
$$

Hence $s(t)<0$, or equivalently, $p_{1}(t)<p_{2}(t)$, for all small $t>0$. In addition, since both $p_{1}(t)$ and $p_{2}(t)$ approach 1 as $t \rightarrow \infty$, we have

$$
\lim _{t \rightarrow \infty} s^{\prime}(t)=\lim _{t \rightarrow \infty}\left(\hat{a}-\hat{b}_{U}+\hat{b}_{U} p_{1}(t)-\left(\bar{a}-\bar{b}_{U}+\bar{b}_{U} p_{2}(t)\right)\right)=\hat{a}-\bar{a}>0
$$

Thus $s(t) \rightarrow \infty$ as $t \rightarrow \infty$, and $p_{1}(t)>p_{2}(t)$ for all $t$ sufficiently large. It follows that $p_{1}(t)$ and $p_{2}(t)$ must coincide at some $t>0$. Let $t^{*}>0$ be the least time at which $p_{1}(t)=p_{2}(t)$, or equivalently, $s(t)=0$. Then

$$
s^{\prime}\left(t^{*}\right)=\hat{a}-\hat{b}_{U}+\hat{b}_{U} p_{1}\left(t^{*}\right)-\left(\bar{a}-\bar{b}_{U}+\bar{b}_{U} p_{1}\left(t^{*}\right)\right)=g_{0}\left(p_{1}\left(t^{*}\right)\right) \geq 0 .
$$

By (28), we see that $p_{1}\left(t^{*}\right)=p_{2}\left(t^{*}\right) \geq \beta_{3}>p_{2}^{*}$. As both $p_{1}$ and $p_{2}$ increase for $t>0$, we have $p_{1}(t)>\beta_{3}$ and $p_{2}(t)>\beta_{3}$ for $t>t^{*}$. Hence $p_{1}$ and $p_{2}$ cannot meet at another time after $t=t^{*}$ since at any possible intersection point it follows from (28) that $s^{\prime}>0$. Note that $p_{1}\left(t^{*}\right)=p_{2}\left(t^{*}\right) \geq \max \left\{\beta_{1}, \beta_{2}, \beta_{3}\right\}$. By using the same argument in the proof of Part (1), we can show that if $p_{2}\left(t_{1}\right) \leq p\left(t_{1}\right) \leq p_{1}\left(t_{1}\right)$ at any $t_{1}>t^{*}$, then this ordering will be maintained for all $t>t_{1}$. In other words, if $p=p(t)$ enters the area $\mathcal{D}_{1}$ at any $t>t^{*}$, then it will stay in this area thereafter. We now show that $p=p(t)$ enters the area $\mathcal{D}_{1}$ when $t$ is sufficiently large. Without loss of generality, we assume that

$$
p\left(t^{*}\right)<p_{1}\left(t^{*}\right)=p_{2}\left(t^{*}\right)
$$

Define

$$
s_{1}(t)=r(t)-r_{2}(t)
$$

Then $s_{1}\left(t^{*}\right)<0$. It remains to show that $s_{1}$ becomes positive at some $t>t^{*}$. Due to the monotonicity of $g_{0}(p)$ in $p$, and $g_{0}(p)=0$ when $p=\beta_{3}$, there is an $\varepsilon>0$ such that $g_{0}(p)>\varepsilon$ when $p>\left(1+\beta_{3}\right) / 2$. Let

$$
\beta_{4}=\max \left\{\frac{1+\beta_{3}}{2}, 1-\frac{\varepsilon}{2 \bar{b}_{U}}, 1-\frac{\varepsilon \bar{T}_{1}}{4 \hat{T}_{2} \bar{b}_{U}}\right\}
$$

Then $\beta_{3}<\beta_{4}<1$. Since $p(t)$ increases and approaches 1 as $t \rightarrow \infty$, there is a unique $t_{4}>0$ such that $\min \left\{p\left(t_{4}\right), p_{1}\left(t_{4}\right), p_{2}\left(t_{4}\right)\right\}=\beta_{4}$.

If the system stays in System (24) at $t>t_{4}$, then

$$
p_{2}(t)-p(t)<1-\left(1-\frac{\varepsilon}{2 \bar{b}_{U}}\right)=\frac{\varepsilon}{2 \bar{b}_{U}},
$$


and therefore

$$
\begin{aligned}
s_{1}^{\prime}(t) & =\hat{a}-\hat{b}_{U}+\hat{b}_{U} p-\left(\bar{a}-\bar{b}_{U}+\bar{b}_{U} p_{2}\right) \\
& >\hat{a}-\hat{b}_{U}+\hat{b}_{U} p-\left(\bar{a}-\bar{b}_{U}+\bar{b}_{U}\left(p+\frac{\varepsilon}{2 \bar{b}_{U}}\right)\right) \\
& =g_{0}(p)-\frac{\varepsilon}{2}>\frac{\varepsilon}{2} .
\end{aligned}
$$

If the system stays in System (25) at $t>t_{4}$, then

$$
s_{1}^{\prime}(t)=\bar{a}-\bar{b}_{U}+\bar{b}_{U} p-\left(\bar{a}-\bar{b}_{U}+\bar{b}_{U} p_{2}\right)>-\bar{b}_{U} \frac{\varepsilon \bar{T}_{1}}{4 \hat{T}_{2} \bar{b}_{U}}=-\frac{\varepsilon \bar{T}_{1}}{4 \hat{T}_{2}}
$$

Suppose that $\left[t_{0}^{*}, t_{0}^{*}+T_{1}\right)$ is a spraying stage and $\left[t_{0}^{*}+T_{1}, t_{0}^{*}+T_{1}+T_{2}\right)$ is a releasing stage with $t_{0}^{*}>t_{4}$. Note that

$$
\begin{aligned}
s_{1}\left(t_{0}^{*}+T_{1}+T_{2}\right) & =s_{1}\left(t_{0}^{*}+T_{1}+T_{2}\right)-s_{1}\left(t_{0}^{*}\right)+s_{1}\left(t_{0}^{*}\right) \\
& \geq \frac{\varepsilon}{2} T_{1}-\frac{\varepsilon \bar{T}_{1}}{4 \hat{T}_{2}} T_{2}+s_{1}\left(t_{0}^{*}\right) \\
& \geq \frac{\varepsilon}{4} T_{1}+s_{1}\left(t_{0}^{*}\right) .
\end{aligned}
$$

It is then clear that $s_{1}(t) \rightarrow \infty$ as $t \rightarrow \infty$, and so $s_{1}(t)$ becomes positive for large $t$. The proof is completed.

\section{Mosquito Control Under the Effect of Heatwave}

In high-temperature condition, mosquitoes may lose Wolbachia according to (Ross et al. 2020, 2017). Let $\mu(0<\mu<1)$ denote the imperfect transmission rate. By making minor changes in (1), we obtain the system

$$
\left\{\begin{array}{l}
\frac{\mathrm{d} x}{\mathrm{~d} t}=b_{I}(1-\mu) x-\delta_{I} x-d x(x+y) \\
\frac{\mathrm{d} y}{\mathrm{~d} t}=b_{I} \mu x+b_{U} y \frac{y}{x+y}-\delta_{U} y-d y(x+y)
\end{array}\right.
$$

If $b_{I}(1-\mu)-\delta_{I}-d \leq 0$, the infected mosquitoes will die out naturally and the mosquito population replacement is going to fail. So we assume

$$
b_{I}(1-\mu)-\delta_{I}-d>0
$$


in the following discussion. Clearly, $E_{1}=\left(0,\left(b_{U}-\delta_{U}\right) / d\right)$ is an infection-free equilibrium and its local stability is determined by the Jacobian of (36),

$$
D F(x, y)=\left(\begin{array}{cc}
b_{I}(1-\mu)-\delta_{I}-2 d x-d y & -d x \\
b_{I} \mu-\frac{b_{U} y^{2}}{(x+y)^{2}}-d y & \frac{b_{U} y(2 x+y)}{(x+y)^{2}}-\delta_{U}-d x-2 d y
\end{array}\right) .
$$

At the infection-free equilibrium point, we have

$$
D F\left(0, \frac{b_{U}-\delta_{U}}{d}\right)=\left(\begin{array}{cc}
b_{I}(1-\mu)-\delta_{I}-b_{U}+\delta_{U} & 0 \\
b_{I} \mu-2 b_{U}+\delta_{U} & \delta_{U}-b_{U}
\end{array}\right) .
$$

This matrix has the eigenvalues $\left(b_{I}-b_{U}\right)+\left(\delta_{U}-\delta_{I}\right)-b_{I} \mu$ and $\delta_{U}-b_{U}$. From (2) and (3), we see that both eigenvalues are negative, and so $E_{1}$ is locally asymptotically stable. To obtain the positive equilibrium in the first quadrant, we solve equations

$$
\left\{\begin{array}{l}
b_{I}(1-\mu) x-\delta_{I} x-d x(x+y)=0 \\
b_{I} \mu x+b_{U} y \frac{y}{x+y}-\delta_{U} y-d y(x+y)=0
\end{array}\right.
$$

The first equation gives

$$
x+y=\left(b_{I}(1-\mu)-\delta_{I}\right) / d:=\kappa_{1} .
$$

Substituting (40) into the second equation yields

$$
A y^{2}-B y+C=0
$$

where

$$
A=b_{U} / \kappa_{1}, \quad B=\delta_{U}+d \kappa_{1}+b_{I} \mu \quad \text { and } \quad C=b_{I} \mu \kappa_{1} .
$$

The discriminant of (41)

$$
\begin{aligned}
\Delta & =B^{2}-4 A C=\left(b_{I} \mu+d \kappa_{1}+\delta_{U}\right)^{2}-4 b_{I} b_{U} \mu \\
& =\left(b_{I} \mu+b_{I}(1-\mu)-\delta_{I}+\delta_{U}\right)^{2}-4 b_{I} b_{U} \mu=\left(b_{I}-\delta_{I}+\delta_{U}\right)^{2}-4 b_{I} b_{U} \mu .
\end{aligned}
$$

Define

$$
\mu^{*}=\left(b_{I}-\delta_{I}+\delta_{U}\right)^{2} / 4 b_{I} b_{U}
$$

It follows from (2) and (3) that $\left(b_{I}-\delta_{I}+\delta_{U}\right)^{2}<4 b_{I} b_{U}$, implying $0<\mu^{*}<1$. If we regard $\Delta$ as a function of $\mu$, then $\Delta(\mu)$ decreases in $\mu$ and $\Delta\left(\mu^{*}\right)=0$. When $\mu>\mu^{*}$, there is no positive equilibrium in the first quadrant and $E_{1}$ is the only stable 

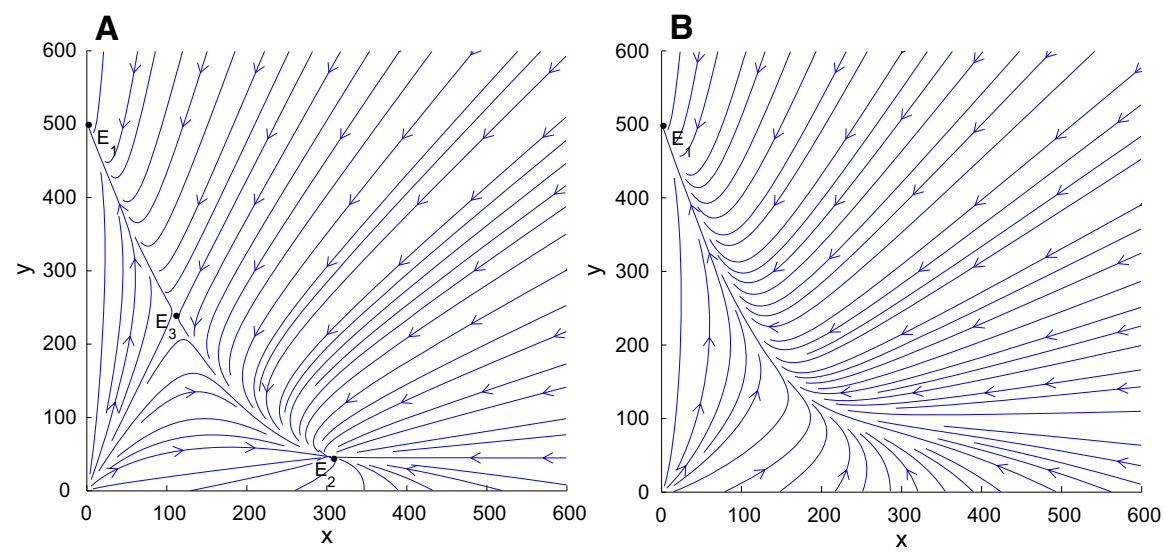

Fig. 5 The vector field direction of system (36). Let $b_{I}=0.45, \mu=0.11, b_{U}=0.55, \delta_{I}=0.05$, $\delta_{U}=0.048, d=0.001$. Panel a shows that system (36) has equilibria $E_{1}, E_{2}$ and $E_{3}$. Compared with system (1), $E_{2}$ becomes an interior equilibrium. Let $\mu$ increase to 0.25 without changing the other parameters. Panel b shows that system (36) only admits an equilibria $E_{1}$

equilibrium. In this case Wolbachia frequency declines to zero. When $\mu<\mu^{*}$, (41) has the solutions

$$
y_{1}=\frac{B-\sqrt{\Delta}}{2 A} \text { and } y_{2}=\frac{B+\sqrt{\Delta}}{2 A},
$$

and both the solutions are nonnegative since $\Delta<B^{2}$. By (40), we obtain the two equilibria

$$
\left(x_{1}, y_{1}\right)=\left(\kappa_{1}-\frac{B-\sqrt{\Delta}}{2 A}, \frac{B-\sqrt{\Delta}}{2 A}\right), \quad\left(x_{2}, y_{2}\right)=\left(\kappa_{1}-\frac{B+\sqrt{\Delta}}{2 A}, \frac{B+\sqrt{\Delta}}{2 A}\right) .
$$

It follows from

$$
\begin{aligned}
y_{2} & =\frac{b_{I}-\delta_{I}+\delta_{U}+\sqrt{\left(b_{I}-\delta_{I}+\delta_{U}\right)^{2}-4 b_{I} b_{U} \mu}}{2 \frac{b_{U}}{\kappa_{1}}} \\
& =\frac{b_{I}(1-\mu)-\delta_{I}}{2 b_{U} d}\left(b_{I}-\delta_{I}+\delta_{U}+\sqrt{\left(b_{I}-\delta_{I}+\delta_{U}\right)^{2}-4 b_{I} b_{U} \mu}\right)
\end{aligned}
$$

that $y_{2}$ decreases in $\mu$. Then $E_{2}\left(x_{1}, y_{1}\right)$ and $E_{3}\left(x_{2}, y_{2}\right)$ stay in the first quadrant when $\mu<\mu^{*}$. As in Farkas and Hinow (2010), we give numerical examples to show the vector field (See Fig. 5).

To offset the negative effect of heatwave on mosquito control, we employ the measures of releasing infected sterile mosquitoes and spraying pesticides to control 
wild mosquitoes as in the previous Section. By taking into account the first measure, we have

$$
\left\{\begin{array}{l}
\frac{\mathrm{d} x}{\mathrm{~d} t}=b_{I}(1-\mu) x-\delta_{I} x-d x(x+y) \\
\frac{\mathrm{d} y}{\mathrm{~d} t}=b_{I} \mu x+b_{U} y \frac{y}{x+y+R(t)}-\delta_{U} y-d y(x+y) .
\end{array}\right.
$$

Theorem 3 Assume that (37) holds and $R(t) \equiv R$. Then the size of wild mosquito population can be suppressed to a level below $\mathcal{S}$ from any positive initial states if the release abundance satisfies the following three conditions:

(i) $R>\frac{4 C b_{U}}{B^{2}}-\kappa_{1}$;

(ii) $R>\min \left\{\frac{b_{U} \kappa_{1}^{2}}{\kappa_{1} B-C}-\kappa_{1}, \frac{2 b_{U} \kappa_{1}}{B}-\kappa_{1}\right\}$;

(iii) $R>\frac{b_{U} \mathcal{S}^{2}}{B \mathcal{S}-C}-\kappa_{1}$ or $R<\frac{2 S b_{U}}{B}-\kappa_{1}$,

where $\kappa_{1}=\left(b_{I}(1-\mu)-\delta_{I}\right) / d, B$ and $C$ are defined in (42).

Proof Similar to the discussion of system (36), the interior equilibrium $(x, y)$ of system (47) is the solution to equations $x+y=\kappa_{1}$ and

$$
A_{R} y^{2}-B y+C=0
$$

where $A_{R}=\frac{b_{U}}{\kappa_{1}+R}, B=\delta_{U}+d \kappa_{1}+b_{I} \mu$ and $C=b_{I} \mu \kappa_{1}$. The discriminant of (48) is

$$
\Delta_{R}=B^{2}-4 A_{R} C=\left(\delta_{U}+d \kappa_{1}+b_{I} \mu\right)^{2}-4 \frac{b_{U}}{\kappa_{1}+R} b_{I} \mu \kappa_{1}
$$

It is easy to verify that $\Delta_{R}$ increases in $R$, and $\Delta_{R}>0$ when condition $(i)$ holds. In this case, (48) has two solutions

$$
y_{1}=\frac{B-\sqrt{\Delta_{R}}}{2 A_{R}} \quad \text { and } \quad y_{2}=\frac{B+\sqrt{\Delta_{R}}}{2 A_{R}}
$$

and both the solutions are nonnegative. From the facts

$$
\begin{aligned}
y_{1} & =\frac{B-\sqrt{B^{2}-4 A_{R} C}}{2 A_{R}}=\frac{2 C}{B+\sqrt{B^{2}-4 A_{R} C}} \\
& =\frac{2 b_{I} \mu \kappa_{1}}{\delta_{U}+d \kappa_{1}+b_{I} \mu+\sqrt{\left(\delta_{U}+d \kappa_{1}+b_{I} \mu\right)^{2}-4 \frac{b_{U}}{\kappa_{1}+R} b_{I} \mu \kappa_{1}}}
\end{aligned}
$$

and

$$
y_{2}=\frac{B+\sqrt{B^{2}-4 A_{R} C}}{2 A_{R}}=\frac{\left(\delta_{U}+d \kappa_{1}+b_{I} \mu\right)+\sqrt{\left(\delta_{U}+d \kappa_{1}+b_{I} \mu\right)^{2}-4 \frac{b_{U}}{\kappa_{1}+R} b_{I} \mu \kappa_{1}}}{\frac{2 b_{U}}{\kappa_{1}+R}}
$$


we see that $y_{1} \rightarrow \frac{C}{B}=\frac{b_{I} \mu \kappa_{1}}{\delta_{U}+d \kappa_{1}+b_{I} \mu}<\kappa_{1}$ and $y_{2} \rightarrow \infty$ when $R \rightarrow \infty$. Then $\left(x_{1}, y_{1}\right)$ stays in the first quadrant and $\left(x_{2}, y_{2}\right)$ stays in the second quadrant when $y_{2}>\kappa_{1}$, which is equivalent to condition ( $i i)$. In this case $\left(x_{1}, y_{1}\right)$ is globally stable and the wild mosquitoes can be suppressed to a level below $\mathcal{S}$ if $y_{1}<\mathcal{S}$, which is equivalent to condition (iii).

Now we consider the measure of spraying pesticides and build the system

$$
\left\{\begin{array}{l}
\frac{\mathrm{d} x}{\mathrm{~d} t}=b_{I}(1-\mu) x-\left(\delta_{I}+\phi_{I}\right) x-d x(x+y), \\
\frac{\mathrm{d} y}{\mathrm{~d} t}=b_{I} \mu x+b_{U} y \frac{y}{x+y}-\left(\delta_{U}+\phi_{U}\right) y-d y(x+y) .
\end{array}\right.
$$

Upon rescaling $\delta_{I}=\delta_{I}+\phi_{I}$ and $\delta_{U}=\delta_{U}+\phi_{U}$, this model is the same as system (36). Here we only discuss the case $\phi_{I}=\phi_{U}$ and the discussion for $\phi_{I} \neq \phi_{U}$ is similar. $\mu^{*}$ defined in (43) is applicable here as $\phi_{I}-\phi_{U}=0$. If $b_{I}(1-\mu)-\delta_{I}-\phi_{I}-d<0$ or $\mu>\mu^{*}$, the infected mosquitoes will die out. Spraying pesticides may suppress wild mosquitoes to a low level, but it takes little use of infected mosquitoes and requires a great deal of pesticides. We next consider the condition

$$
\mu<\mu^{*} \quad \text { and } \quad b_{I}(1-\mu)-\delta_{I}-\phi-d>0
$$

Define

$$
A=\frac{d b_{U}}{b_{I}(1-\mu)-\delta_{I}-\phi}, \quad B=\delta_{U}+b_{I}-\delta_{I}, \quad C=\frac{b_{I} \mu}{d}\left(b_{I}(1-\mu)-\delta_{I}-\phi\right) .
$$

Theorem 4 Let $\phi$ be the excess death rate of both infected and uninfected mosquitoes caused by pesticides. Suppose that (51) holds. Then the size of wild mosquito population can be suppressed to a level below $\mathcal{S}$ if $y(0) / x(0)<y_{2} / x_{2}$ and

$$
\phi>b_{I}(1-\mu)-\delta_{I}-\max \left\{\frac{2 d b_{U} \mathcal{S}}{B-\sqrt{B^{2}-4 b_{I} b_{U} \mu}}, \frac{2 d \mathcal{S} b_{U}}{B}\right\}
$$

where

$$
x_{2}=\frac{b_{I}(1-\mu)-\delta_{I}-\phi}{d}-\frac{B+\sqrt{B^{2}-4 A C}}{2 A}, \quad y_{2}=\frac{B+\sqrt{B^{2}-4 A C}}{2 A},
$$

Proof When $\mu<\mu^{*}$, system (50) admits two interior equilibria $E_{2}\left(x_{1}, y_{1}\right)$ and $E_{3}\left(x_{2}, y_{2}\right)$ given by (45) if $\delta_{I}$ and $\delta_{U}$ are replaced by $\delta_{I}+\phi$ and $\delta_{U}+\phi$, respectively. As shown in Fig. 5A, $E_{2}\left(x_{1}, y_{1}\right)$ is locally stable while $E_{3}\left(x_{2}, y_{2}\right)$ is an unstable saddle point. Then the wild mosquitoes can be suppressed to the below $\mathcal{S}$ if the following two conditions hold: $(i) E_{2}$ stays below $y=\mathcal{S}$; ( $\left.i i\right)$ the initial state lies in 
the basin of attraction of $E_{2}$. It follows from (44) that condition $(i)$ is equivalent to $\frac{B-\sqrt{B^{2}-4 A C}}{2 A}<\mathcal{S}$, which implies

$$
\begin{aligned}
\phi & >\min \left\{b_{I}(1-\mu)-\delta_{I}-\frac{2 d b_{U} \mathcal{S}}{B-\sqrt{B^{2}-4 b_{I} b_{U} \mu}}, b_{I}(1-\mu)-\delta_{I}-\frac{2 d \mathcal{S} b_{U}}{B}\right\} \\
& =b_{I}(1-\mu)-\delta_{I}-\max \left\{\frac{2 d b_{U} \mathcal{S}}{B-\sqrt{B^{2}-4 b_{I} b_{U} \mu}}, \frac{2 d \mathcal{S} b_{U}}{B}\right\}
\end{aligned}
$$

Let $p(t)$ denote the infection density in system (50). Then

$$
\begin{aligned}
\frac{\mathrm{d} p(t)}{\mathrm{d} t} & =\frac{x^{\prime} y-x y^{\prime}}{(x+y)^{2}} \\
& =\frac{x y}{(x+y)^{2}}\left(b_{I}(1-\mu)-\delta_{I}-b_{I} \mu \frac{x}{y}-b_{U} \frac{y}{x+y}+\delta_{U}\right) \\
& =\frac{x y}{(x+y)^{2}}\left(\left(b_{I}(1-\mu)-\delta_{I}+\delta_{U}\right)-\left(b_{I} \mu \frac{x}{y}+b_{U} \frac{1}{x / y+1}\right)\right)
\end{aligned}
$$

Define

$$
f(r)=b_{I} \mu r+\frac{b_{U}}{r+1}
$$

Since $f^{\prime}(r)=b_{I} \mu-b_{U} /(1+r)^{2}$, we see that $f^{\prime}(r)$ increases in $r$ when $r>0$ and $f^{\prime}\left(r^{*}\right)=0$ with $r^{*}=\sqrt{\frac{b_{U}}{b_{I} \mu}}-1$. Then $f(r)$ decreases in $r$ when $0 \leq r \leq r^{*}$ and increases in $r$ when $r \geq r^{*}$. In addition, $f(0)=b_{U}$ and $f(r) \rightarrow \infty$ when $r \rightarrow \infty$. On the other hand, $E_{2}\left(x_{1}, y_{1}\right)$ and $E_{3}\left(x_{2}, y_{2}\right)$ are two interior equilibria, so $r_{1}=x_{1} / y_{1}$ and $r_{2}=x_{2} / y_{2}$ are two roots to equation

$$
\left(b_{I}(1-\mu)-\delta_{I}+\delta_{U}\right)-f(r)=0 .
$$

It follows from (52) that $p(t)$ decreases in $t$ if $x(t) / y(t)<x_{2} / y_{2}$ or $x(t) / y(t)>x_{1} / y_{1}$, and increases in $t$ if $x_{2} / y_{2}<x(t) / y(t)<x_{1} / y_{1}$. Thus $y=\frac{y_{2}}{x_{2}} x$ is the separatrix of the basins of attraction of $E_{2}$ and $E_{3}$, implying that condition ( $i i$ ) holds if the initial state $(x(0), y(0))$ satisfies $y(0) / x(0)<y_{2} / x_{2}$.

Finally, we consider applying the above two measures together to suppress wild mosquitoes under the effect of heatwave. The integrated model is given by

$$
\left\{\begin{array}{l}
\frac{\mathrm{d} x}{\mathrm{~d} t}=b_{I}(1-\mu) x-\left(\delta_{I}+\phi\right) x-d x(x+y), \\
\frac{\mathrm{d} y}{\mathrm{~d} t}=b_{I} \mu x+b_{U} y \frac{y}{x+y+R(t)}-\left(\delta_{U}+\phi\right) y-d y(x+y) .
\end{array}\right.
$$

By combining Theorem 3 and 4, we obtain the following result. 
Remark 3.1 Let $\phi$ be the excess death rate of both infected and uninfected mosquitoes caused by pesticides and $R(t) \equiv R$. Suppose that (51) holds. Then the size of wild mosquito population can be suppressed to a level below the safe threshold $\mathcal{S}$ if the release abundance and pesticide effect satisfy

$$
\begin{aligned}
& \text { (i) } B^{2}-4 A_{R} C>0, \quad \text { (ii) } \frac{B+\sqrt{B^{2}-4 A_{R} C}}{2 A_{R}}>\kappa_{1} \text { and } \text { (iii) } \frac{B-\sqrt{B^{2}-4 A_{R} C}}{2 A_{R}}<\mathcal{S}, \\
& \text { where } \kappa_{1}=\frac{b_{I}(1-\mu)-\delta_{I}-\phi}{d}, \quad A_{R}=\frac{b_{U}}{\kappa_{1}+R}, \quad B=\delta_{U}+b_{I}-\delta_{I} \quad \text { and } C=b_{I} \mu \kappa_{1} .
\end{aligned}
$$

The proof follows from Theorem 3 and 4 directly.

\section{Discussion}

Some mosquito-borne diseases, such as dengue fever, occur periodically and are triggered by imported patients in some sub-tropical areas. It is essential to take emergency measures when the dengue cases are large. As there is no vaccine or effective medication available, eliminating the transmission vector has been the most effective method. The traditional measure uses pesticides, which kills mosquitoes quickly but cannot suppress mosquitoes for a long time due to insecticide resistance. One promising complementary method is to use incompatible insect technique (IIT) by releasing Wolbachia-infected mosquitoes into wild mosquito populations, which has been proven to be a novel and environmental-friendly way for mosquito control. Many interesting models of difference or differential equations have been developed to investigate the dynamic behavior of wild mosquito populations based on IIT, see (Hu et al. 2019; Huang et al. 2018; Keeling et al. 2003; Shi and Yu 2020; Yu and Zheng 2019; Zhang et al. 2020; Zheng et al. 2014) and the references therein. The radiation-based sterile insect technique (SIT) uses radiation to sterilize male mosquitoes, leading irradiated males that are unable to produce offspring after mating with wild females. Mathematical models for studying the suppression effects on SIT have provided applicable guidance (Cai et al. 2014; Li and Yuan 2015; Yu 2020; Yu and Li 2019, 2020). Several interesting mathematical models have been developed to deal with mosquito control via a combination of Wolbachia and insecticides (Li and Liu 2020; Qu et al. 2018; Zheng et al. 2018). In this work, we introduced population suppression measures, releasing infected sterile mosquitoes and spraying pesticides, into population replacement model to discuss the mosquito control.

In Sect. 2, we provided the estimations of wild mosquito abundance or infection density in normal environment under different control measures. Mosquito control is always affected by environmental conditions. In Hu et al. (2019), we considered the case that female mosquitoes lay diapause eggs in cold season in a sub-tropical area. In this work, we consider the opposite extreme case that infected mosquitoes may lose Wolbachia in a high-temperature season (Ross et al. 2020, 2017). Under the effect of heatwave, the offspring of infected females lose Wolbachia infection 
A

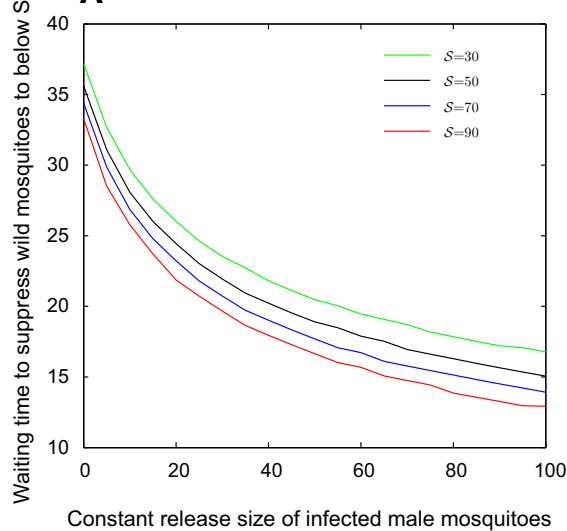

B

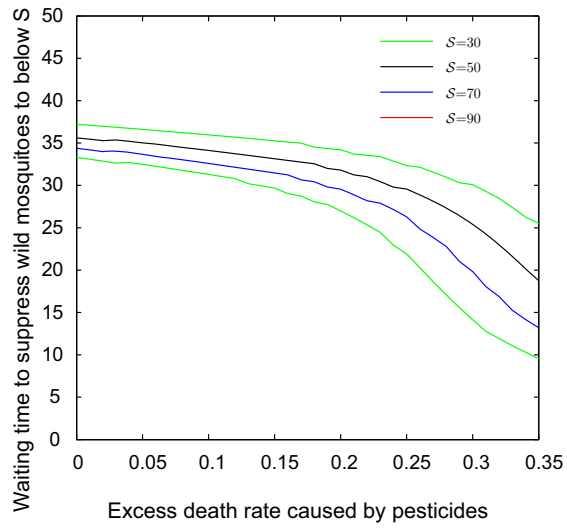

Fig. 6 The waiting times to suppress wild mosquito abundance to a level below $\mathcal{S}$. Let $b_{I}=0.45, b_{U}=$ $0.55, \delta_{I}=0.05, \delta_{U}=0.048, d=0.001$ and $\left(x_{0}, y_{0}\right)=(200,800)$. Panel a shows the waiting times to suppress wild mosquito abundance to a level below $\mathcal{S}$ under different releasing amounts, and panel b shows the waiting times under different pesticide effects (Color figure online)
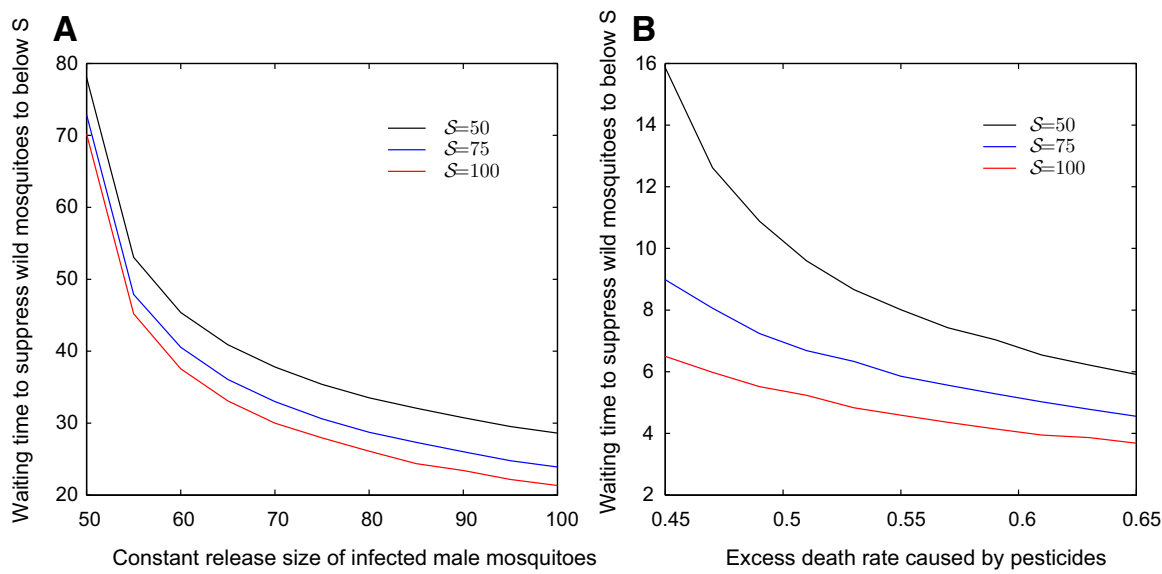

Fig. 7 The waiting times to suppress wild mosquito abundance to a level below $\mathcal{S}$. Let the parameters and initial state be the same as in Fig. 6 and set $\mu=0.1$. Panel a shows the waiting times to suppress wild mosquito abundance to a level below $\mathcal{S}$ under different releasing amounts, and panel b shows the waiting times under different pesticide effects (Color figure online)

with positive leakage rate $\mu$. We discussed how to offset the effect of the heatwave in sect. 3. For example, we consider the case where $b_{I}=0.45, b_{U}=0.55, \delta_{I}=$ $0.05, \delta_{U}=0.048, d=0.001$ and the initial state $\left(x_{0}, y_{0}\right)=(200,800)$. The wild mosquitoes can be replaced by infected mosquitoes totally without control measures in model (1). However, by releasing infected mosquitoes or spraying pesticides we can greatly increase the replacement speed. Figure 6 shows the waiting times to suppress wild mosquito abundance to a level below $\mathcal{S}$ with the measure of release infected mosquitoes (Figure 6A) or spraying pesticides (Figure 6B). 
Let the parameters be the same as in Fig. 6 and set $\mu=0.1$. Then infected mosquitoes will die out in competing with wild uninfected mosquitoes in model (36). In this case, the extra measures must be taken to ensure the successful suppression of wild mosquitoes. Figure 7 shows the waiting time to suppress wild mosquito abundance to a level below $\mathcal{S}$ under the measures of releasing infected mosquitoes (Fig. 7A) or spraying pesticides (Fig. 7B). With the help of Figs. 6 and 7, we can choose the strategy to suppress wild mosquitoes based on the actual conditions and requirement.

Recently, by releasing Wolbachia-infected mosquitoes twice a week for three years (Zheng et al. 2019), our team shows that combining incompatible and sterile insect techniques (IIT-SIT) enables near elimination of the populations of Aedes albopictus in Shazai island, Guangzhou. To prevent bites from the female mosquitoes mixed in the released males, measures should be taken to reduce or even eliminate the number of females in each release. For the case that all the subsequent released mosquitoes are male, we can consider sex structure in (4) by changing $R(t)$ to $2 R(t)$, and the related discussions are nearly the same. We believe that the combination of spraying pesticides and releasing Wolbachia-infected mosquitoes can play an important role in mosquito control. In this work, we studied the mosquito replacement in a normal environmental condition and high temperature condition with heatwaves, respectively. However, the actual habitat conditions are changeable and difficult to be described by two simple stable conditions. Furthermore, the mosquito population can be also affected by many other factors, such as migration (Schmidt and Barton 2017) and urbanization process ( $\mathrm{Li}$ and Kamara 2014). The specific effects of insecticides are also complicated. In the future work, we will explore more information of mosquito growth and make good use of the advantages of the two measures to formulate mathematical models to study mosquito control.

Acknowledgements We thank the two reviewers very much for their valuable comments and suggestions. This work was supported by National Natural Science Foundation of China (11631005, 11971127).

Open Access This article is licensed under a Creative Commons Attribution 4.0 International License, which permits use, sharing, adaptation, distribution and reproduction in any medium or format, as long as you give appropriate credit to the original author(s) and the source, provide a link to the Creative Commons licence, and indicate if changes were made. The images or other third party material in this article are included in the article's Creative Commons licence, unless indicated otherwise in a credit line to the material. If material is not included in the article's Creative Commons licence and your intended use is not permitted by statutory regulation or exceeds the permitted use, you will need to obtain permission directly from the copyright holder. To view a copy of this licence, visit http://creativecommons.org/licenses/by/4.0/.

\section{References}

Bian G, Xu Y, Lu P et al (2010) The endosymbiotic bacterium Wolbachia induces resistance to dengue virus in Aedes aegypti. PLoS Pathog 6:e1000833

Cai L, Ai S, Li J (2014) Dynamics of mosquitoes populations with different strategies for releasing sterile mosquitoes. SIAM J Appl Math 74(6):1786-1809

Callaway E (2020) The mosquito strategy that could eliminate dengue. Nat News. https://doi.org/10.1038/ d41586-020-02492-1

Caspari E, Watson GS (1959) On the evolutionary importance of cytoplasmic sterility in mosquitoes. Evolution 13:568-570 
Duan J, Lin L, Cai S, Liu W, Yi J, Lu W, Yin W (2009) Study on the stepwise responses for risk categories for dengue fever vector. Chinese J Vector Biol Cont 20(1):51-54

Dutra HLC, Rocha MN, Dias FBS, Mansur SB, Caragata EP, Moreira LA (2016) Wolbachia blocks currently circulating Zika virus isolates in brazilian Aedes aegypti mosquitoes. Cell Host Microbe 19(6):771774

Farkas JZ, Hinow P (2010) Structured and unstructured continuous models for Wolbachia infections. Bull Math Biol 72:2067-2088

Hu L, Huang M, Tang M, Yu J, Zheng B (2015) Wolbachia spread dynamics in stochastic environments. Theor Popul Biol 106:32-44

Hu L, Huang M, Tang M, Yu J, Zheng B (2019) Wolbachia spread dynamics in multi-regimes of environmental conditions. J Theor Biol 462:247-258

Hu L, Tang M, Wu Z, Xi Z, Yu J (2019) The threshold infection level for Wolbachia invasion in random environments. J Diff Equ 266:4377-4393

Huang M, Lou J, Hu L, Zheng B, Yu J (2018) Assessing the efficiency of Wolbachia driven Aedes mosquito suppression by delay differential equations. J Theor Biol 440:1-11

Huang M, Tang M, Yu J, Zheng B (2020) A stage structured model of delay differential equations for Aedes mosquito population suppression. Discret Contin Dyn Syst A 40(6):3467-3484

Keeling MJ, Jiggins FM, Read JM (2003) The invasion and coexistence of competing Wolbachia strains. Heredity 91:382-388

Kyle JL, Harris E (2008) Global spread and persistence of dengue. Annu Rev Microbiol 62:71-92

Laven H (1956) Cytoplasmic inheritance in Culex. Nature 177:141-142

Li J, Yuan Z (2015) Modelling releases of sterile mosquitoes with different strategies. J Biol Dyn 9(1):1-14

Li Y, Liu X (2020) Modeling and control of mosquito-borne diseases with Wolbachia and insecticides. Theor Popul Biol 132:82-91

Li Y, Kamara F et al (2014) Urbanization increases Aedes albopictus larval habitats and accelerates mosquito development and survivorship. PLos Negl Trop Dis 8(1):e3301

McMeniman CJ, Lane RV, Cass BN et al (2009) Stable introduction of a life-shortening Wolbachia infection into the mosquito Aedes aegypti. Science 323:141-144

Ooi EE, Goh KT, Gubler DJ (2006) Dengue prevention and 35 years of vector control in Singapore. Emerg Infect Dis 12:887-893

Qu Z, Xue L, Hyman JM (2018) Modeling the transmission of Wolbachia in mosquitoes for controlling mosquito-borne diseases. Siam J Appl Math 78(2):826-852

Rasmussen SA, Jamieson DJ, Honein MA, Petersen LR (2016) Zika virus and birth defects-reviewing the evidence for causality. N Engl J Med 374:1981-1987

Ross PA, Axford JK, Yang Q et al (2020) Heatwaves cause fluctuations in wMel Wolbachia densities and frequencies in Aedes aegypti. PLoS Negl Trop Dis 14(1):e0007958

Ross PA, Wiwatanaratanabutr I, Axford JK, White VL, Endersby-Harshman NM, Hoffmann AA (2017) Wolbachia infections in Aedes aegypti differ markedly in their response to cyclical heat stress. PLoS Pathog 13(1):e1006006

Ryan PA, Turley AP, Wilson G et al (2019) Establishment of wMel Wolbachia in Aedes aegypti mosquitoes and reduction of local dengue transmission in Cairns and surrounding locations in northern Queensland. Australia. Gates Open Res 3:1547

Schmidt TL, Barton NH et al (2017) Local introduction and heterogeneous spatial spread of denguesuppressing Wolbachia through an urban population of Aedes aegypti. PLoS Biol 15(5):e2001894

Shi Y, Yu J (2020) Wolbachia infection enhancing and decaying domains in mosquito population based on discrete models. J Biol Dyn 14(1):679-695

Walker T, Johnson PH, Moreira LA et al (2011) The wMel Wolbachia strain blocks dengue and invades caged Aedes aegypti populations. Nature 476(7361):450-453

Weeks AR, Reynolds KT, Hoffmann AA (2002) Wolbachia dynamics and host effects: what has (and has not) been demonstrated? Trends Ecol Evol 17(6):257-262

Yeap HL, Mee P, Walker T et al (2011) Dynamics of the "popcorn" Wolbachia infection in outbred Aedes aegypti informs prospects for mosquito vector control. Genetics 187:583-595

Yu J (2020) Existence and stability of a unique and exact two periodic orbits for an interactive wild and sterile mosquito model. J Diff Equ 269(12):10395-10415

Yu J (2018) Modeling mosquito population suppression based on delay differential equations. SIAM J Appl Math 78(6):3168-3187 
Yu J, Li J (2019) Dynamics of interactive wild and sterile mosquitoes with time delay. J Biol Dyn 13(1):606620

Yu J, Li J (2020) Global asymptotic stability in an interactive wild and sterile mosquito model. J Diff Equ 269(7):6193-6215

Yu J, Zheng B (2019) Modeling Wolbachia infection in mosquito population via discrete dynamical models. J Diff Equ Appl 25:1549-1567

Zhang D, Zheng X, Xi Z, Bourtzis K, Gilles JR (2015) Combining the sterile insect technique with the incompatible insect technique: I-impact of Wolbachia infection on the fitness of triple-and doubleinfected strains of Aedes albopictus. PLoS ONE 10(4):e0121126

Zhang X, Liu Q, Zhu H (2020) Modeling and dynamics of Wolbachia-infected male releases and mating competition on mosquito control. J Math Biol 81:243-276

Zheng B, Tang M, Yu J (2014) Modeling Wolbachia spread in mosquitoes through delay differential equations. SIAM J Appl Math 74:743-770

Zheng B, Tang M, Yu J, Qiu J (2018) Wolbachia spreading dynamics in mosquitoes with imperfect maternal transmission. J Math Biol 76:235-263

Zheng B, Yu J, Xi Z, Tang M (2018) The annual abundance of dengue and Zika vector Aedes albopictus and its stubbornness to suppression. Ecol Model 387:38-48

Zheng X, Zhang D et al (2019) Incompatible and sterile insect techniques combined eliminate mosquitoes. Nature 572:56-61

Publisher's Note Springer Nature remains neutral with regard to jurisdictional claims in published maps and institutional affiliations. 